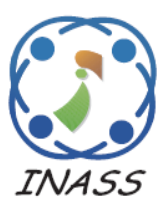

\title{
Optimal View Point Selection in Direct Volume Rendering for 3D Visualization
}

\author{
Gunasekaran Ganesan $^{1 *} \quad$ Venkatesan MeenakshiSundram $^{2}$ \\ ${ }^{I}$ School of Information Technology and Engineering, \\ Vellore Institute of Technology, Vellore, India \\ *Corresponding author's Email: gunasekarang865@gmail.com
}

\begin{abstract}
D visualization is the recent research need to be able to view the model from various angles so as to see data that is obscured by other data. While visualizing the data, view point selection is one of the major challenge which define the viewpoint information to select best viewpoints. This paper introduces teaching learning opacity based optimization (TLOO) and Kalman filter for selecting optimal viewpoint. The structural features of 3D volume dataset such as opacity and luminance is extracted for selecting optimal viewpoint position from that structural features. TLOO optimization algorithm identifies the voxels and standard information using view point evolution function. Then, kalman filter steers the optimization process with the help of cost function. The proposed flow of the technique is simple and very effective for selecting optimal viewpoints in 3D images. Computational complexity and the quality metrics are analyzed that provided maximum output efficiency.
\end{abstract}

Keywords: 3D visualization, Volume rendering, View point selection, Kalman filter.

\section{Introduction}

The volume rendering is generally used to render the $2 \mathrm{D}$ image projected into $3 \mathrm{D}$ data sets by a $3 \mathrm{D}$ scalar field. The volume rendering technique is carried out to find a surface features accuracy [1]. The volume rendering is generally classified in to indirect and direct classes. The direct class render volumetrically information to establish 2D image [2]. Indirect class render is managed faster than direct class rendering which offers to reduce standard 3D rendering method modification [3]. The condition in rendering indicates a unit area, volume which may be a 3D cubic factor array. To develop a 3D data set volume rendering technique is one of the optimized method which is developed here [4]. For imaging development in medical field, rendering is carried out by direct class rendering and its approach is said to be successful and hard to find current approach is insufficient. The images are rendered to realistic by employing mathematical method to get an optimistic image. These visualization application and technique offer to develop a real time data [5]. The transfer functions (TF) is to use best technique for DVR operation. The transfer functions are using real time in computerized tomography data sets [6]. The 3D volume rendering align data's based on transfer function that is used to design function domain to luminance and opacity. In medical field 3D visualization which will monitor patient's capability to generate visualization by point of dynamic discussion, modifying transparency, offering clipping planes and tissue colour [7].

Engineers and scientist become assistant their knowledge on information transmission, mobile device and remote rendering programming in order to recognize this practicality [8] [9]. A 3D interpretation carries contusion structural data and linked formation and $2 \mathrm{D}$ analysis requirements to find out local data at intervals single slice images [10]. DVR acquired point out to be superior that time this context and compared to indirect approaches, whatever consider polygonal models [11]. It supports constant image information partition using TFs' that assign darkness and colour to each data value. To avoid time absorbing recover in a particular active function 
stages. Respectively, aggregate manipulation and high-feature production are valuable choice to employing during a clinical setting [12]. Being imaging operation 3D image has become a popular approaches, as well as computer supported recognition, image-guided radiotherapy and surgery [13]. For this activity using segmentation, that grants volumes of interest (VOI's) and visual separation. 3D substance is suitable for great demonstrations like volumetric and multi-modality, polygonal and attribute (often get to obtain) from data sets [14]. As every good of information set on its own different image technique, this heterogeneity to render numerous objects within information explanation cause it strong as a single rendering pipeline [15]. The concurrent rendering isn't that easy once there's a change in data structure between them with multiple uniform datasets.

However, serious loss is that systems availability, whatever contributes exact graphics volume for clinical application [17] [18]. High-end servers, it suggests basic suggestion that to handle enormous scientific information sets, high-performance IO bandwidth and calculation power [19]. Again, systems are fast growing as resolved rendering competence and calculation. The aim is to correctly determine boundary of prostate on rendered images, and employing CT data sets is to improve opacity transfer functions to analyze prostate from normal viewing adaptation [20]. The significant goal is to have a computer justify 'good' viewpoint(s) to customer. We have consecutive two (not necessarily disjoint) instructions for determining a valid viewpoint: (i) a viewpoint is valid if voxel with high consequence factors have high visibilities. (ii) $\mathrm{A}$ viewpoint is good if volumetric data set projection has a high information quantity. The optimization problem is to select optimal viewpoint from search space using advanced techniques [21].

In this paper the proposed non-conventional optimization method TLOO provides accurate results regardless of voxel's opacity and luminance. This algorithm is based on teaching learning technique which does not require specific controlling parameters. Hence it provides global solution with high consistency and less computational effort. This algorithm is efficient and it takes less number of iterations over PSO and SFLA. In our proposed study improved a fundamental part in viewpoint selection, as it's an additional uncertain viewpoint evaluations place and mainly enhance searching process capability.

The rest of the paper is organized as follows. Section 2 describes the related works and section 3 describes the proposed approach of view point selection. The implementation results and conclusions are represented in section 4 and 5 respectively.

\section{Related work}

Alper M. Selver [22] employing brush lets increase volumetrically information (i.e., domain of TF) that symbolize high-frequency and every low uneven structures are completely various circumference in transform domain. The developed brush let coefficients also used for application of preferred portion and reorganization of volume for non-linear handling. By using atlas and machine learning based technique, transfer function is difficult, slow and unintuitive. Hence there is a need of simplest transfer function for making the volume data visible. Using well-known $\mathrm{TF}$ requirement technique's applications to intestinal information sets achieve with MR, CT, and PET shows that prepared volume improvement efficiently creates higher standard of 3D rendering Kyoko Hasegawa et al. [23]. Sorting of transparent object in unity is a problem associated with the method of transparent-rendering.

It illustrates slice images using colour and opacity with non-uniform opacity design the same as volume rendering. In medical, for analysis, inner threedimensional design transparent surface visual image is mostly used Dong-Joon Kim et al. [24]. During polygons should be arranged on vision line, such visualization classically includes use of polygonal shape graphics. However, for large-scale information that are categorize by enlarged computation time. Consequently, doesn't need arranging is planned as a transparent-rendering method for particle-based volume rendering. They offer $3 \mathrm{D}$-fused pictures to show performance of planned method, as well as volume-slice and slice-slice pictures, for medical volumetrically knowledge. In this the rendering pipelines used should be restructured and it needs an additional algorithm for restructuring. The conclusion show that achievement of their work is suitable in cases within empire of particle is larger than that of cell Youn Hyun Jung et al. [25].

This research presents a novel different medical objects intermixing technique for fusion rendering whereas protecting real-time performance. For current applications within medical system various 3D image objects has been increasingly needed. Because of information structure or no consistency in information explanation, it's tough to with efficiency render multiple medical objects in high quality. Finally, they developed a slab-based visibility intermixing technique with recently estimated rendering pipeline supported effective in-slab and z- 
slab visibility interpolation. By introducing a dualmodality positron emission imaging, in a physical related case supported by CT and CT (PET-CT) represent pathophysiological do with PET.

To allow visual image for three-dimensional volume rendering approaches and a two-dimensional slice of interest (SOI) from PET meet with DVR (DVR) from CT. Correlated with various slice-based and their prior methods, it shows developments with visualization approach. Then prompt clinical analysis of their visual image during a PET-CT analysis sequence of patients with non-small cell lung cancer and developed cartilage volume and thickness quantified using an edge detection based. However, the time taken for selecting depth parameter, occlusion information leads to the removal of valuable contextual cues. An interactive segmentation technique from knee joint magnetic resonance images (MRI), and joint visualized in 3D M. S. Mallikarjunaswamy et al. [26].

To reduce amount of voxel volume of interest (VOI) process method used and treated in 3D rendering of articular cartilage. As compared with physical and different semi-automatic strategies decrease processing time. Using Bland-Altman plots as compared with physical strategies handled of thickness and volume calculation was evaluated. The contour VOI must be defined for each orthogonal direction. VOI will vary with time; hence it is difficult to define the same VOI at all time. The selected viewpoints are needed to be optimized for best visualization. PSO and SFLA are the popular optimization algorithms for view point selection in volume rendering. Due to the simplicity of TLOO, it is proposed in 3D dataset visualization.

\section{Proposed methodology for locating optimal view using DVR}

The main criterion discussed on this paper is evolution of an advanced technique for choosing optimal viewpoint used in DVR. The optimization is carried out to find viewpoint entropy value of different structural features at different angles. To develop an optimal view point, measure 3D image entropy rate which offer a structural features such as opacity and luminance of projected view of input image. These parameters are tried to indicate voxel and structural data within that that image data set. These data sets are being used by TLOO for optimal view point estimation. Thus optimal view is selected and it is subsequent to maximum number of entropy value. By improving line opacity and surface, optimal viewpoint can be located. The proposed algorithm can locate optimal viewpoint by logically and automatically. For cost function estimation, proposed approach using Kalman filter as a medium for reduction of cost function in optimization process.

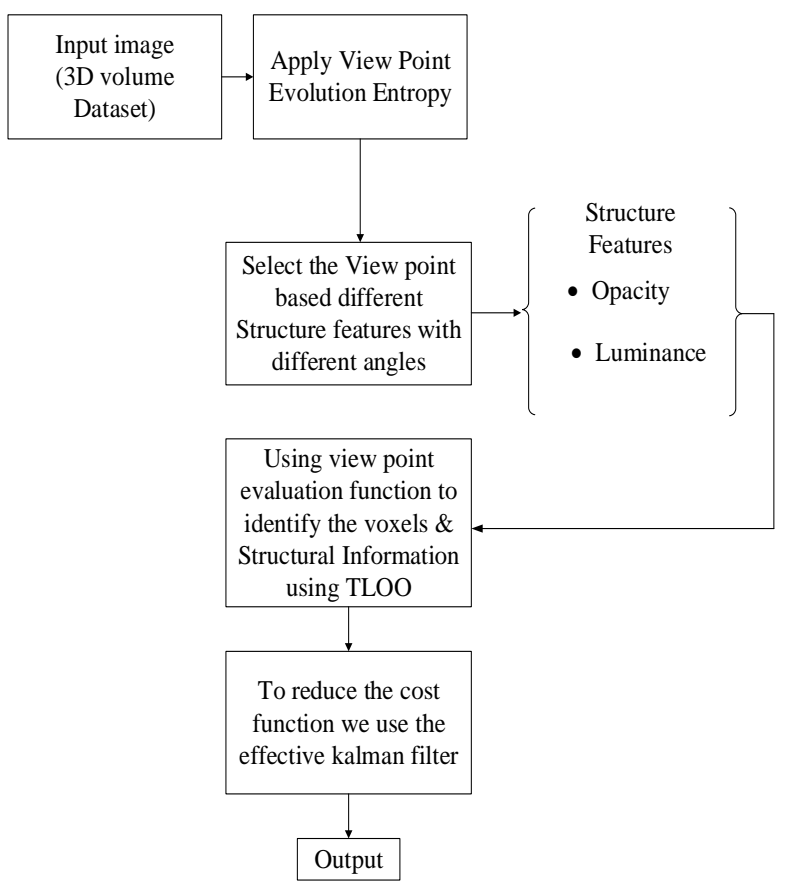

Figure.1 Block diagram for proposed work

Graphical representation of proposed work diagram is given in Fig. 1 which shows input parameter as a 3D volume data set. The image is said to find optimal view point evolution entropy based on different structural features such as opacity and luminance. The view point evaluation is carried out with structural data by TLOO algorithm and final approach of optimization is to reduce cost function which can be estimated by using Kalman filter to proposed algorithm by which it completes the proposed work.

\subsection{Viewpoint evolution entropy}

The viewpoint evolution entropy is evaluated by using computerized thermography data sets. The main problem affecting viewpoint selection is to choosing optimal viewpoint. In volume rendering process image visibility is determined by voxel which is used for opacity and luminance estimation. The reason for aspects are opacity and luminance are moved for attain optimal viewpoint. For larger structural information user recommends the rendering viewpoint for a processing. The viewpoint entropy of a given view can be executed from following equation. 


$$
H(V)=\sum_{z=0}^{M-1}\left[\begin{array}{l}
\left(\begin{array}{c}
w_{a z} \cdot \alpha_{z}+w_{k z} \cdot k_{z} \\
\sum_{q=0}^{M-1}\left(w_{a q} \cdot \alpha_{q}+w_{k q} \cdot k_{q}\right)
\end{array}\right) * \\
\left.\log _{2} \frac{w_{a z} \cdot \alpha_{z}+w_{k z} \cdot k_{z}}{\sum_{q=0}^{M-1}\left(w_{a q} \cdot \alpha_{q}+w_{k q} \cdot k_{q}\right)}\right)
\end{array}\right]
$$

Where, $M$ means number of pixels in rendered image, $\alpha_{z}$ and $k_{z}$ are taken as opacity and luminance of the pixel i. The normalized orthogonal gradient attitude of opacity and luminance are taken as $\mathrm{w}_{\mathrm{az}}$ and $\mathrm{w}_{\mathrm{kz}}$, they describe structure information within 3D volume data set.

\subsection{TLOO based optimized view point selection}

The viewpoint entropy is selected and best point is calculated, by after issues are figured out over view sphere, then TLOO algorithm is applied to calculate most simple set of candidate views. The population (learner) which is taken as entropy and structure features i.e. opacity and luminance which act as instructor. TLOO is a population based algorithmic function where entropy is taken as input parameter. The initialization starts by applying population of entropy which is generally consider as learners. The learner phase is considered as population phase and structure features are considered as instruction and learning effect which is identical to the fitness value in optimization. In TLOO algorithm, in this main aspect is to find out error less entropy. In proposed work, best solution is considered as instructor. TLOO is categorized in to two phases one is teaching phase and another one is learning phase. In teaching phase is trained by an instructor and learning phase is trained by intercommunication with in the learner phase. Both phase functions are described in Fig. 2 below.

\subsubsection{Teaching phase}

In this phase, learning is done by taking ideas from learners (entropy) and optimizes mean results. Let assume $\mathrm{N}$ as number of learners and $\mathrm{r}_{\mathrm{z}}$ as number of learners in population range, $\mathrm{z}=1,2 \ldots \mathrm{n}$. The mean result of learners be $\mathrm{N}_{\mathrm{q}}$ in this range $\mathrm{q}=1,2 \ldots$ $\mathrm{n}$. The learners mean result can be calculated from following equation,

$$
N_{q}=r_{z}\left(Y_{q}^{z b e s t}-T_{p} N_{q}\right)
$$

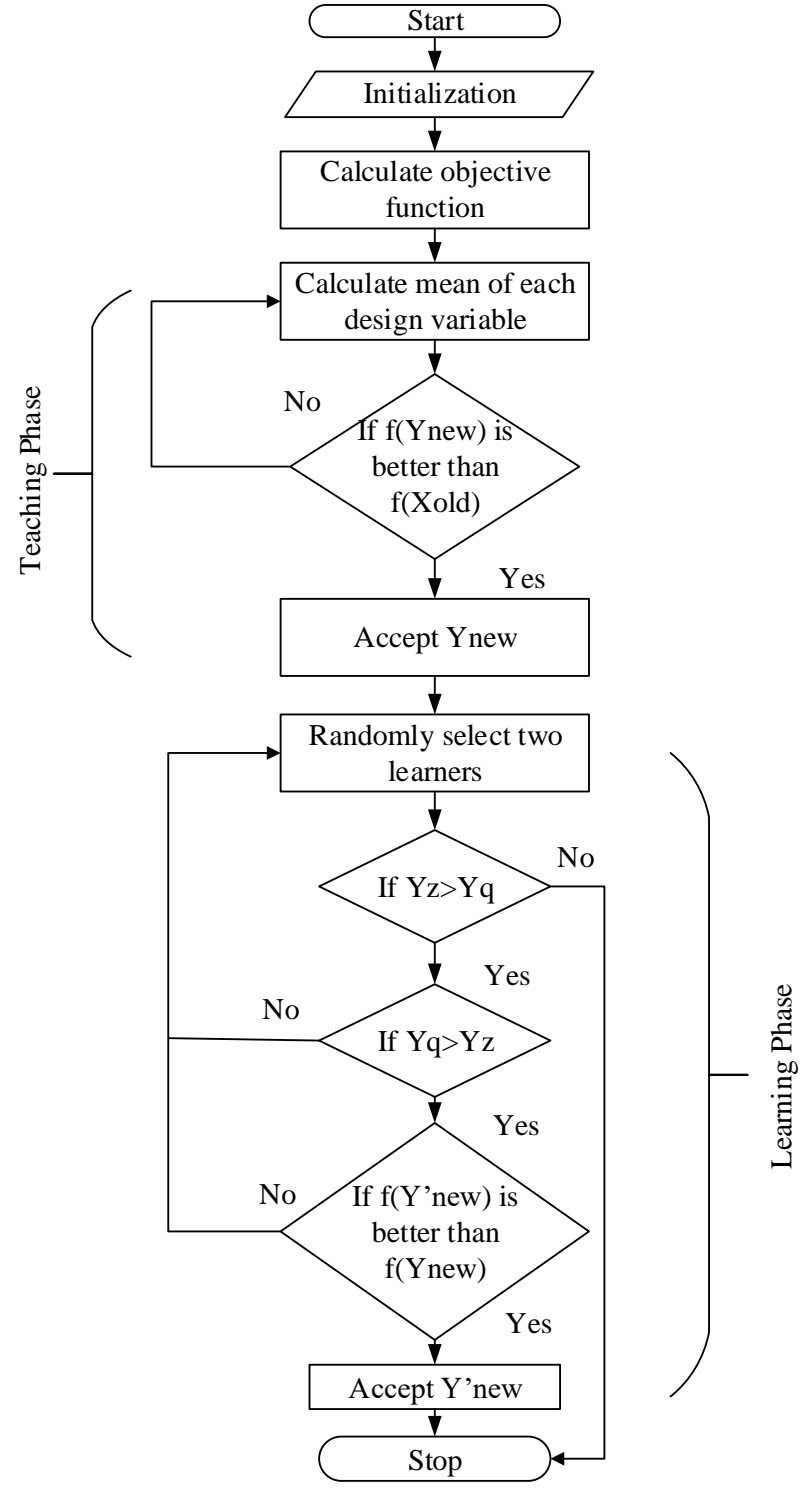

Figure.2 Flow Chart for TLOO Algorithm

Where $\mathrm{Y}_{\mathrm{q}}^{\text {zbest }}$ denotes optimal best learner in this range of $q, T_{p}$ means teaching factor in learner phase is randomly chosen in this range $(1,2)$ and $r_{z}$ means random number in this range from 0 to 1 . The training factor $T_{f}$ can be calculated from following equation below.

$$
T_{p}=\operatorname{round}[1+\operatorname{rand}(0,1)\{2-1\}]
$$

$\mathrm{T}_{\mathrm{p}}$, is not a norm of the TLOO algorithm. The value of $\mathrm{T}_{\mathrm{p}}$ is not disposed as a source of algorithm and its value is randomly determined by Eq. (2). By equation (2) $\mathrm{N}_{\mathrm{q}}$ is modify in teacher phase according to below expression.

$$
Y_{\text {new }}=Y_{\text {old }}+N_{q}
$$


Where $Y_{\text {old }}$ represents the existing solution, $Y_{\text {new }}$ denote renovate value of $Y_{\text {old. }} Y_{\text {new }}$, is accepted, if it support better outcome.

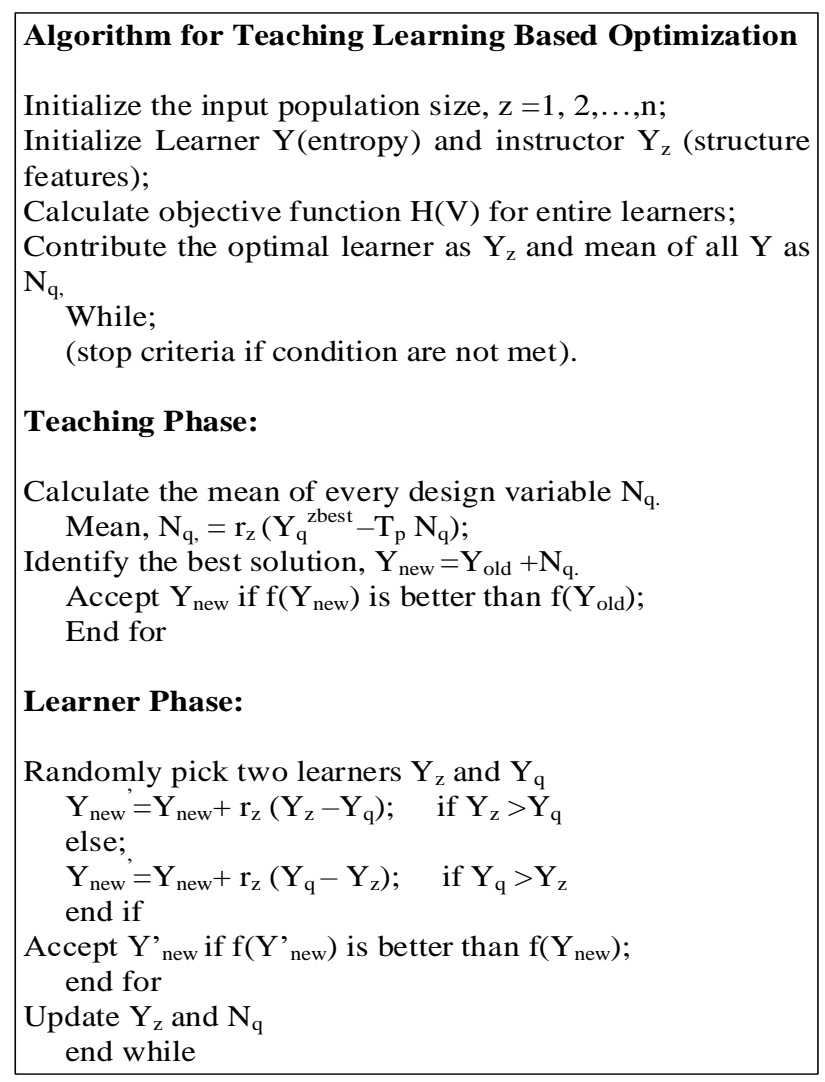

Figure.3 TLOO Algorithm

\subsubsection{Learner phase}

The learning phase provides instruction to learners by understanding between learners. The learners gain idea by communicate with similar other learners. Thus learner selects new data if learner has more idea than other learner. Select two learners randomly, $\mathrm{Y}_{\mathrm{z}}$ and $\mathrm{Y}_{\mathrm{q}}$ are pick such that $\mathrm{z} \neq \mathrm{q}$ (where, $Y_{z}$ and $Y_{q}$ are modify values of $Y_{z}$ and $N_{q}$ properly at the end of teacher phase).

$$
\begin{array}{cc}
Y_{\text {new }}^{\prime}=Y_{\text {new }}+r_{z}\left(Y_{z}-Y_{q}\right) & \text { if } Y_{z}>Y_{q} \\
Y_{\text {new }}^{\prime}=Y_{\text {new }}+r_{z}\left(Y_{q}-Y_{z}\right) & \text { if } Y_{q}>Y_{z}
\end{array}
$$

$\mathrm{Y}_{\text {new }}$, value is approved, if it contributes a better function value. The value of $r_{z}$ is the random number in the range of $(0,1)$. The pseudo code for the teaching learning based optimization algorithm is given in Fig. 3.

\subsection{Kalman filter (EKF)}

The Kalman Filter employ predictor corrector algorithm to measure current state variables, along with their inconsistency. The Kalman filter used to control noisy system. There are two types of relation for Kalman filter such as prediction and correction. The prediction equation anticipates what the current state is located on previous state and commanded action. The second set of equations known as update equations view at your origin of sensors, how much you recognize every sensor, and how much you gain your overall state estimate. This filter works by anticipating present state uses prediction equations follow by examine how well at a job it did predicting by using update equations. This operation is repeated to update the present state.

\subsubsection{Prediction equations}

i) Compute the state and measurement equations:

$$
X_{t}=A_{t} y_{t-1}+B_{t} z_{t}
$$

Where, $X_{t}$ denote Kalman filter with true state time interval between $t$ and $t-1, A_{t}$ means state transition model with previous state time interval $y_{t-1}$ and $B_{t}$ means input control model with controlling vector at time interval $\mathrm{z}_{\mathrm{t}}, \mathrm{z}_{\mathrm{t}}$ denotes the measurement variance matrix.

ii) Compute error covariance matrices:

$$
R_{t-1}=A_{t} R_{t-1} A_{t}^{s}+D_{t}
$$

Where, $R_{t-1}$ represents the state variance matrix, $A_{t}$ is the state transition model, $\mathrm{A}_{t}{ }^{\mathrm{s}}$ is the transpose of $\mathrm{A}_{\mathrm{t}}$, $D_{t}$ is process variance matrix.

\subsubsection{Updating}

iii) Kalman gain calculation:

$$
\begin{aligned}
& G_{k}=R_{t-1} J^{i} O_{m}{ }^{-1} \\
& O_{m}=J_{t} R_{t-1} J_{t}^{i}+L_{t}
\end{aligned}
$$

Where $\mathrm{R}_{\mathrm{t}-1}$ means state variance matrix, $\mathrm{J}$ denotes the observation matrices, $\mathrm{O}_{\mathrm{m}}^{-1}$ is the inverse of residual covariance, $\mathrm{L}_{\mathrm{t}}$ is measurement variable.

iv) Kalman gain updating:

$$
\begin{aligned}
X_{t} & =X_{t-1}+F\left(I_{t}\right) \\
I_{t} & =c-J X_{t-1}
\end{aligned}
$$


Table 1. Performance correlation

\begin{tabular}{|c|c|c|c|c|}
\hline Method & SNR & PSNR & MSE & $\begin{array}{c}\text { Computat } \\
\text { ional cost }\end{array}$ \\
\hline PSO & 26.526 & 28.222 & 45.330 & 28.879 \\
\hline SFLA & 23.876 & 26.8113 & 54.396 & 28.182 \\
\hline TLBO & 38.265 & 39.859 & 40.797 & 27.985 \\
\hline
\end{tabular}

Where, $\mathrm{c}$ is measurement matrix state, $\mathrm{F}$ denote Kalman gain, $\mathrm{I}_{\mathrm{t}}$ is residual covariance, $\mathrm{J}$ denotes the observation matrices.

v) Covariance update:

$$
R X_{t}=(1-F J) R_{t-1}
$$

Where, $\mathrm{F}$ denotes the Kalman gain, $\mathrm{J}$ denotes the observation matrices and $\mathrm{R}_{\mathrm{t}-1}$ represents the state variance matrix.

\section{Experimental results and analysis}

The proposed work is carried out in an advanced stage that supports MRI, CT, PET, VOREEN which includes such formats like JPEG, BMP etc. A PET scan is carried out on mouse raw data which is captured on Quad Hidac small animal PET scanner with a data set of $150 \times 150 \times 276$ voxel with 16 bits is taken for the execution of outputs. The execution of proposed work is carried out in Mat Lab platform by enhancing convergence rate, SNR, PSNR, MSE and lowering the view point difficulty. The performance evaluation among proposed and existing algorithm are explained in below Table 1 .

In Fig. 4, Kalman filter output reorganize the image after viewing all optimal points. The image is reorganized from $3 \mathrm{D}$ raw data to $2 \mathrm{D}$ image using proposed algorithm. By analysing algorithmic function proposed algorithm offer better performance when it is compared with particle swarm optimization (PSO) [27] and shuffled frog leaping algorithm (SFLA) [28].

The histogram design for 2D image is shown in Fig. 5 for proposed work with actual PSO and SFLA. The $\mathrm{x}$-axis of the histogram plot represent the intensity and $y$-axis representing the range of pixel values.

Signal to noise ratio (SNR): The signal to noise ratio is defined as mean signal ratio to average standard deviation. The SNR can be evaluated by following equation.

$$
S N R=10 \log \left(\delta^{2} / \lambda^{2}\right)
$$

Where $\delta$ is mean value, $\lambda$ is standard deviation. Mean and standard deviation estimated using following equation:

$$
\begin{aligned}
& \delta=\frac{1}{n} \sum_{i=1}^{n} v_{i} \\
& \lambda=\sqrt{\frac{\sum(v-\delta)^{2}}{C}}
\end{aligned}
$$

Where, $v_{i}$ represent a variable at the $i^{\text {th }}$ index of a dataset, $n$ represents the total number of variables, and $\mathrm{C}$ is total count of numbers in dataset.

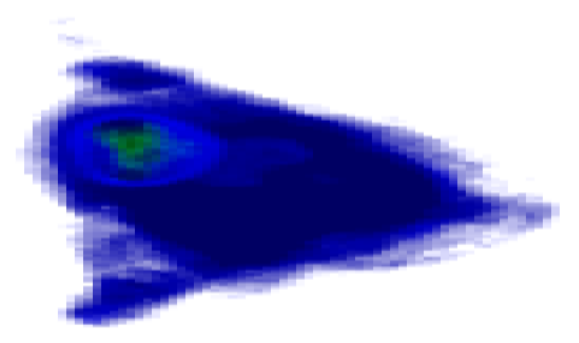

(a)

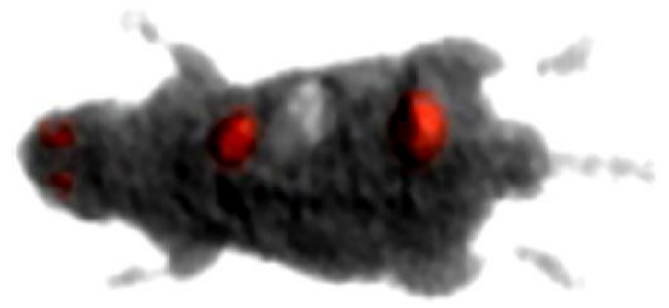

(b)

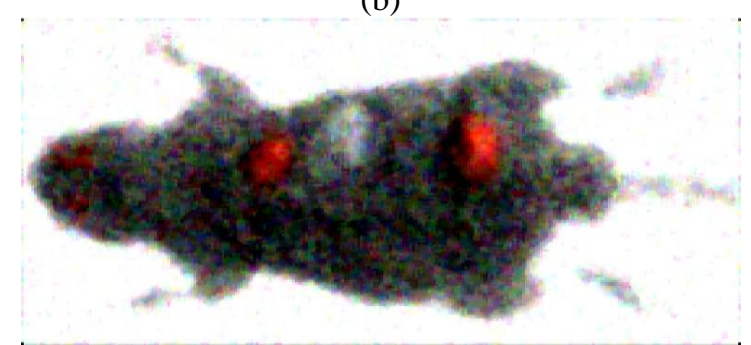

(c)

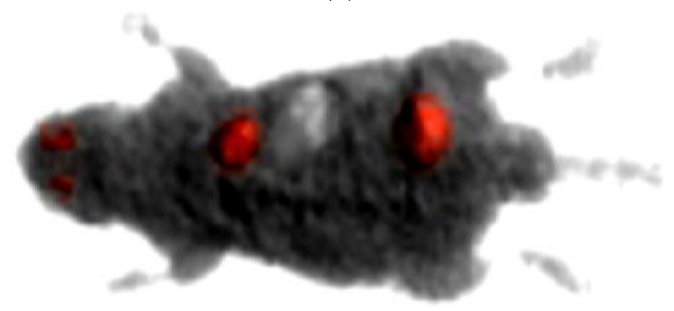

(d)

Figure.4 Approximate outcome for reconstructed 2D image from 3D raw data: (a) 3D raw data (b) proposed, (c) PSO and (d) SFLA algorithms 


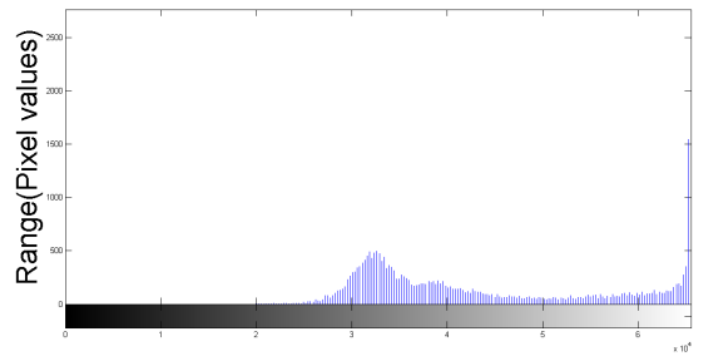

(a)

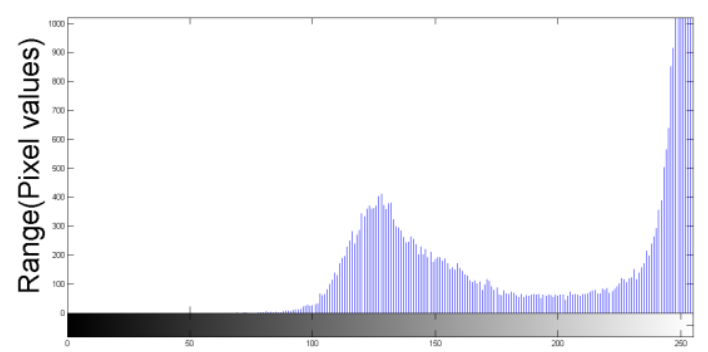

(b)

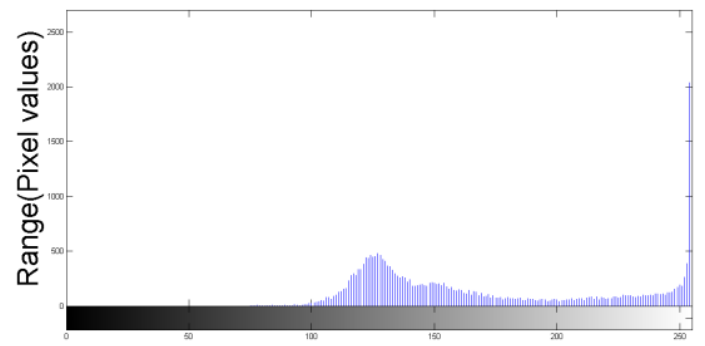

(c)

Figure.5 Approximate Histogram design for the 2D image: (a) Proposed, (b) PSO and (c) SFLA

Mean square error (MSE): The mean square error means loss carried out in variance of estimation which is derived from following equation.

$$
M S E=\frac{1}{m n} \sum_{i=1}^{m} \sum_{k=1}^{n}\left[T(i, k)-T^{\prime}(i, k)\right]
$$

Here $T(i, k)$ express original pixel value, and $T^{\prime}(i, j)$ denoted as decoded pixel value.

\section{Peak signal to noise ratio (PSNR):}

$$
P S N R=10 \log _{10} \frac{255^{2}}{M S E}
$$

Where, MSE represents the mean square error.

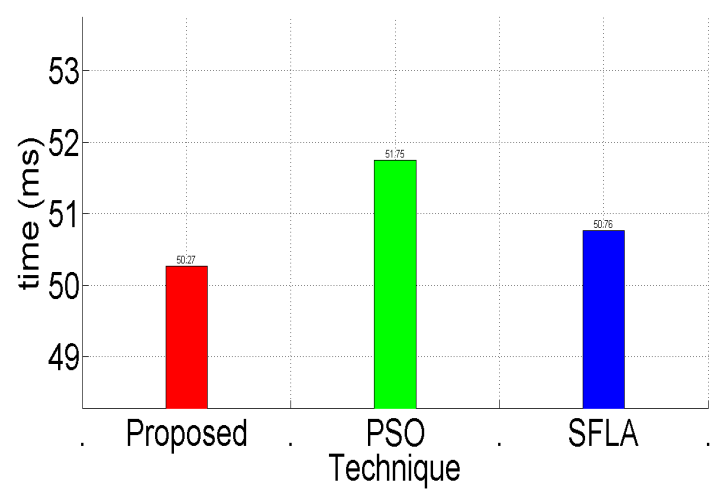

Figure.6 Correlation of Computational cost

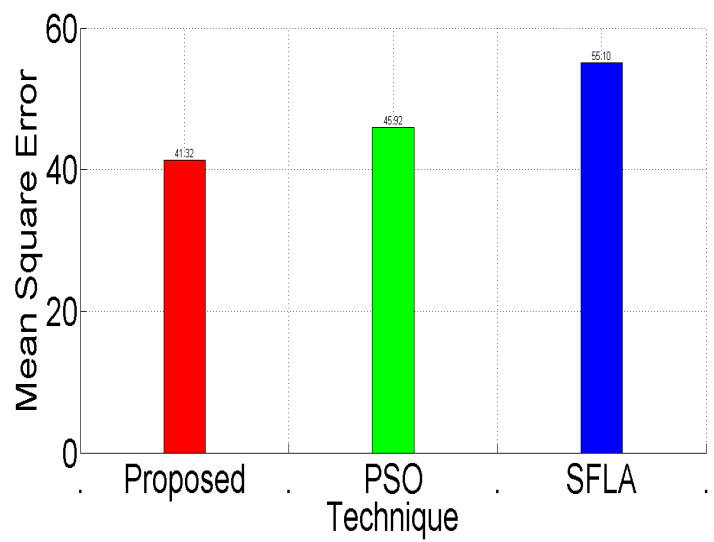

Figure.7 Relation of MSE

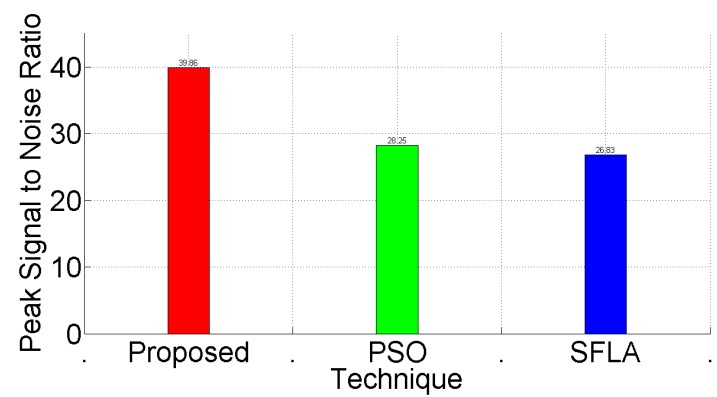

Figure.8 Relation of PSNR

The computational cost of proposed TLOO algorithm is increased with $0.46 \mathrm{~ms}$ than existing techniques in Fig. 6. It shows the implementation efficiency of proposed TLOO. The PSO and SFLA produced MSE value 55.20 and 46.00 respectively. But for the proposed method it was reduced to 41.40 and it was shown in Fig.7.

Figures 8 and 9 show the increased performance for SNR and PSNR. The convergence rate of proposed method is heavily increased in the Shannon range between $10^{-1.2}$ and $10^{-1.1}$. For the existing algorithms, there is no such improvement in Fig. 10. 


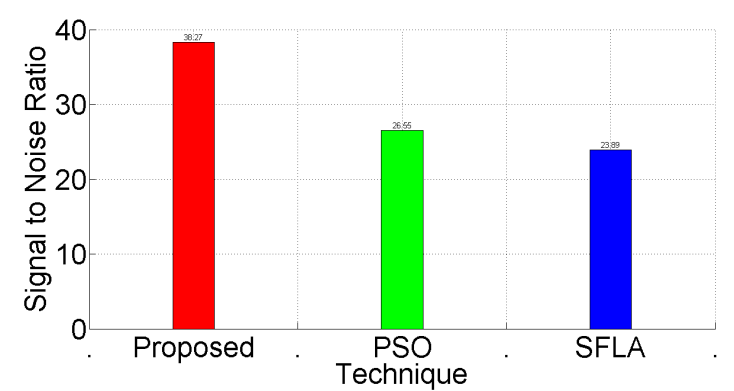

Figure.9 Relation of SNR

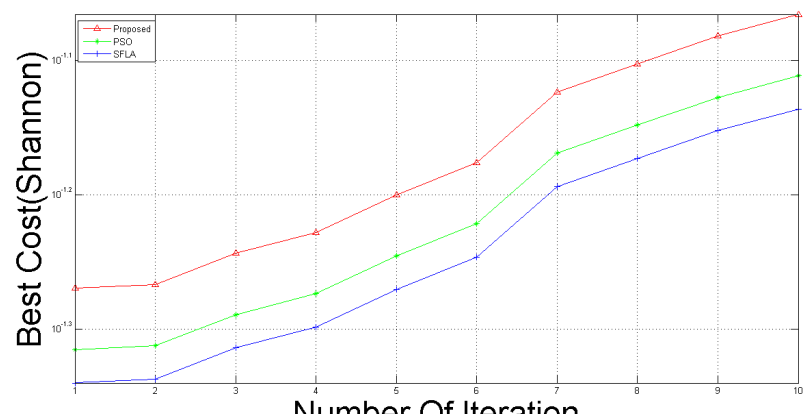

Figure.10 Convergence chart comparative analysis

The traditional PSO and SFLA algorithms are easily fall into local optimum in high dimensional space and has a low convergence rate in the iterative process. But in case of TLOO, the convergence rate is higher than other approaches and it does not require algorithm-specific parameters. Hence, the computational complexity of proposed method is low when it is applied to solve the high dimensional and complex problems. The developed method is recommend improved performance correlated with actual PSO and SFLA in correlation with performance metrics such as computational cost, SNR, PSNR, MSE and convergence rate shows that Figs. 6 to 10 .

\section{Conclusion}

In this research developed a new approach for selecting optimal view point in DVR. Improved TLOO technique is used this paper for optimal view point evaluation. The view point evolution entropy used here to find entropy from the 3D dataset which will be beneficial for providing structural information and voxel effects within 3D volume data. The maximum entropy value is taken from this evaluation and it uses optimize lines and surface opacity for getting optimized view point. TLOO algorithm is utilized in this process for automatically choosing best view. For cost function evaluation, Kalman filter is induced in view point selection. The performance of proposed work is estimated in terms of convergence rate, SNR, PSNR and MSE. The performance results show the improved performance of volume rendering in 3D image with reduced computational complexity. The future work focuses on incorporating personalized user requirements into view point selection algorithm and providing best viewpoint selection for time varying dataset.

\section{References}

[1] C. Lundström, P. Ljung, A. Persson, and A. Ynnerman, "Uncertainty visualization in medical volume rendering using probabilistic animation", IEEE transactions on visualization and computer graphics, Vol.13, No.6, pp.16481655, 2007.

[2] K. Engel, P. Hastreiter, B. Tomandl, K. Eberhardt, and T. Ertl, "Combining local and remote visualization techniques for interactive volume rendering in medical applications", In: Proc. of the Visualization 2000, pp.449-452, 2000.

[3] T. Hachaj, "Real time exploration and management of large medical volumetric datasets on small mobile devices - evaluation of remote volume rendering approach", International Journal of Information Management, Vol.34, No.3, pp.336-343, 2014.

[4] B.A. Jani, J.S. Irick, C. Pelizzari, "Opacity Transfer Function Optimization for Volumerendered Computed Tomography Images of the Prostate 1", Academic radiology, Vol.12, No.6, pp.761-770, 2005.

[5] G. Gunasekaran, and B.K. Ray, "Three Dimensional Visualization for Fast Display of Vector Fields Using Illuminated Stream Lines", ARPN Journal of Engineering and Applied Sciences, Vol.9, No.3, pp.237-243, 2014.

[6] G. Gunasekaran, and M. Venkatesan, "Review on Visualization Techniques for Medical Data and Its Application", Research Journal of Pharmaceutical, Biological and Chemical Sciences, Vol.7, No.3, pp.169-173, 2016.

[7] J. Kim, W. Cai, S. Eberl, and D. Feng, "Realtime volume rendering visualization of dualmodality PET/CT images with interactive fuzzy thresholding segmentation", IEEE Transactions on Information Technology in Biomedicine, Vol.11, No.2, pp.161-169, 2007.

[8] J. Beyer, M. Hadwiger, S. Wolfsberger, and K. Bühler, "High-quality multimodal volume rendering for preoperative planning of neurosurgical interventions", IEEE 
Transactions on Visualization and Computer Graphics, Vol.13, No.6, pp.1696-1703, 2007.

[9] T. Iwahashi, K. Shino, K. Nakata, H. Otsubo, T. Suzuki, H. Amano, and N. Nakamura, "Direct anterior cruciate ligament insertion to the femur assessed by histology and 3-dimensional volume-rendered computed tomography", Arthroscopy: The Journal of Arthroscopic \& Related Surgery, Vol.26, No.9, pp.13-20, 2010.

[10] D. Jönsson, M. Falk, and A. Ynnerman, "Intuitive exploration of volumetric data using dynamic galleries", IEEE Transactions on Visualization and Computer graphics, Vol.22, No.1, pp.896-905, 2016.

[11] J. Kniss, G. Kindlmann, and C. Hansen, "Multidimensional transfer functions for interactive volume rendering", IEEE Transactions on Visualization and Computer Graphics, Vol.8, No.3, pp.270-285, 2002.

[12] H.A.E. Fettouh, R.B. Herts, T. Nimeh, L.S. Wirth, A. Caplin, M. Sands, P.A. Ramani, J. Kaouk, A.D. Goldfarb, and Inderbir S. Gill, "Prospective comparison of 3-dimensional volume rendered computerized tomography and conventional renal arteriography for surgical planning in patients undergoing laparoscopic donor nephrectomy", The Journal of urology, Vol.170, No.1, pp.57-60, 2003.

[13] Z. Jiawan, S. Jizhou, S. Zhigang, and W. Zunce, "Moment based transfer function design for volume rendering", In: Proc. of the International Conference on Computational Science and Its Applications, pp.266-274, 2003.

[14] S. Mori, T. Takaya, M. Kinugasa, T. Ito, S. Takamine, S. Fujiwara, T. Nishii, "Threedimensional quantification and visualization of aortic calcification by multidetector-row computed tomography: a simple approach using a volume-rendering method", Atherosclerosis, Vol.239, No.2, pp.622-628, 2015.

[15] Y. Sato, C.F. Westin, A. Bhalerao, S. Nakajima, N. Shiraga, S. Tamura, and R. Kikinis, "Tissue classification based on 3D local intensity structures for volume rendering", IEEE Transactions on Visualization and Computer Graphics, Vol.6, No.2, pp.160-180, 2000.

[16] F.R. Spaide, "Volume-rendered optical coherence tomography of retinal vein occlusion pilot study", American Journal of Ophthalmology, Vol.31, No.165, pp.133-144, 2016.

[17] L. Wang, Y. Zhao, K. Mueller, and A. Kaufman, "The magic volume lens: An interactive focus+ context technique for volume rendering", In:
Proc. of the Visualization, 2005, VIS 05, IEEE, pp.367-374, 2005.

[18] M. Yamura, T. Hirai, Y. Korogi, I. Ikushima, Y. Yamashita, and S. Oishi, "Pseudostenosis in vessels adjacent to intracranial aneurysms on volume-rendered 3D angiograms: A phantom study1", Academic Radiology, Vol.12, No.3, 305-308, 2005.

[19] J. Yao, D.N. Masani, Q.L. Cao, P. Nikuta, and G.N. Pandian, "Clinical application of transthoracic volume-rendered threedimensional echocardiography in the assessment of mitral regurgitation", The American Journal of Cardiology, Vol.82, No.2, pp.189-196, 1998.

[20] Y. Zheng, D.F. Yu, J.G. Zhao, Y.L. Wu, and B. Zheng, "3D printout models vs. 3D-rendered images: which is better for preoperative planning", Journal of Surgical Education, Vol.73, No.3, pp.518-523, 2016.

[21] P.J. Schulze, and U. Lang, "The parallelized perspective shear-warp algorithm for volume rendering", Parallel Computing, Vol.29, No.3, pp.339-354, 2003.

[22] M.A. Selver, "Exploring brushlet based 3D textures in transfer function specification for direct volume rendering of abdominal organs", IEEE Transactions on Visualization and Computer Graphics, Vol.21, No.2, pp.174-187, 2015.

[23] K. Hasegawa, Y. Fujimoto, R. Xu, T. Tateyama, Yen-Wei Chen, and Satoshi Tanaka, "Fused Visualization with Non-uniform Transparent Surface for Volumetric Data Using Stochastic Point-Based Rendering", In: Proc. of the Innovation in Medicine and Healthcare, 2016, Springer International Publishing, pp.237-246, 2016.

[24] D.J. Kim, B. Kim, J. Lee, J. Shin, K.W. Kim, and Y.G. Shin, "High-quality slab-based intermixing method for fusion rendering of multiple medical objects", Computer Methods and Programs in Biomedicine, 123, pp. 27-42, 2016.

[25] Y.H. Jung, J. Kim, Dagan Feng, and Michael Fulham, "Occlusion and Slice-based Volume Rendering Augmentation for PET-CT", IEEE Journal of Biomedical and Health Informatics, No.99, 2016.

[26] M. S. Mallikarjunaswamy, S.M. Holi, and R. Raman. "Quantification and 3D Visualization of Articular Cartilage of Knee Joint Using Image Processing Techniques", Computational Intelligence in Data Mining-Volume, Vol. 2, pp.417-425, 2015. 
[27] Y. Wang, and Y. Zheng, "Viewpoint selection using PSO algorithms for volume rendering", In: Proc. of the Computer and Computational Sciences, IMSCCS 2007, Second International Multi-Symposiums on IEEE, pp. 286-291, 2007.
[28] Y. Zhang, and B. Wang, "Optimal viewpoint selection for volume rendering based on shuffled frog leaping algorithm", In: Proc. of the Progress in Informatics and Computing (PIC), IEEE International Conference, Vol. 2, pp. 706709, 2010. 